\title{
Neue Strahlenschutzverordnung - Ultraschall in der Schwangerschaft ist gefährlich? Eine nicht haltbare Feststellung
}

Zum 31.12.2018 ist die neue Strahlenschutzverordnung in Kraft getreten. Diese beschäftigt sich mit der Anwendung ionisierender Strahlung. Im Artikel 4 geht es dann erstmals um die Anwendung sogenannter nichtionisierender Strahlen und Wellen am Menschen, wie z. B. Laser und dem weit harmloseren Ultraschall. Gegenstand ist die Anwendung des Ultraschalls zu kosmetischen und nichtmedizinischen Zwecken im Rahmen gewerblicher und sonstiger wirtschaftlicher Unternehmungen.

Der konkrete Wortlaut des $\S 10$ lautet allerdings: Anwendung von Ultraschall an einer schwangeren Person. Bei der Anwendung von Ultraschallgeräten zu nichtmedizinischen Zwecken darf ein Fötus nicht exponiert werden.

Dazu ist von Seiten der DEGUM Folgendes festzustellen: Die Durchführung des diagnostischen Ultraschalls in Deutschland ist durch einen Arztvorbehalt gekennzeichnet, d. h. es wird immer nur ein Ultraschall zu medizinischen Zwecken durchgeführt. Dem entspricht bereits eine Stellungnahme der DEGUM, die im Jahr 2012 im Deutschen Ärzteblatt publiziert wurde.

In der Begründung zu $§ 10$ wird der medizinisch indizierte Ultraschall nach Mutterschaftsrichtlinien einschließlich aller eventuell zusätzlich notwendigen Ultraschalluntersuchungen ausdrücklich vom Verbot ausgenommen und nur das sogenannte „Baby-Fernsehen“, bei dem Ultraschall des Fötus im Sinne einer Eventveranstaltung eingesetzt wird, erwähnt. Fachlich betrachtet werden beide Untersuchungen mit den gleichen Ultraschallgeräten durchgeführt, sodass sich die Frage stellt - wo ist der Unterschied?
Dieser Paragraf setzt unbegründet eine Methode unter einen gesundheitsgefährdenden Generalverdacht, der durch keine einzige Studie erhärtet werden kann.

Die Fragen, die durch die pauschale, unserer Meinung nach wissenschaftlich nicht haltbare Begründung von $\S 10$ SSV aufgeworfen werden, können wie folgt beantwortet werden:

Gibt es eine Gesundheitsbelastung des Fötus durch Ultraschall?

\section{Antwort: Nein!}

Es gibt trotz jahrzehntelanger intensivster Forschungsarbeit nach wie vor keine Studienergebnisse, die auf irgendeine ultraschallinduzierte „Gesundheitsbelastung“ des Fötus hindeuten. Studien (Duggan 1994, Helmy 2015) haben gezeigt, dass eine theoretische Temperaturerhöhung in hohem Maße von der Intensität des abgegebenen Schalls abhängig ist und unter Verwendung eines heute üblichen Leistungsniveaus deutlich unter dem Temperaturanstieg liegt, der durch Fieber oder starke körperliche Aktivität ausgelöst wird!

Lediglich eine spezielle und nur nach strenger Indikationsstellung eingesetzte Betriebsart zur Messung von Strömungsgeschwindigkeiten, der sogenannte PW-Doppler, geht mit einer höheren Schallemission einher und könnte nach mehrminütiger (!) kontinuierlicher Beschallung theoretisch einen Temperaturanstieg von mehreren Grad Celsius herbeiführen. Aus diesem Grunde wird diese Betriebsart nur zur Ursachenforschung bei bekannten Wachstumsstörungen und einigen weiteren Auffälligkeiten eingesetzt, wobei die Beschallungszeit üblicherweise auf wenige Sekunden beschränkt bleibt. Darüber hinaus wird dem Untersucher auf dem Bildschirm die zu erwartende Temperaturerhöhung kontinuierlich angezeigt. Des Weiteren werden dabei meist Gefäße untersucht, die außerhalb des Fötus liegen, wie die mütterliche Gebärmutterarterie oder die Nabelschnur am Ansatz an dem Mutterkuchen. In seltenen Fällen werden bei sehr speziellen Fragestellungen direkt fetale Gefäße untersucht, die sich aber meist im Bereich parenchymatöser Organe wie z. B. Leber oder Lunge befinden. In sehr wenigen Ausnahmefällen - bei klarer Indikationsstellung - wird das fetale Gehirn untersucht.

\section{Beeinflusst der Ultraschall die fetale Hirn- entwicklung?}

\section{Antwort: Nein!}

Es gibt keine eindeutige Datenlage, die eine Beeinflussung der fetalen Hirnentwicklung belegt. Der von einigen Forschern in den USA gemutmaßte Zusammenhang zwischen Ultraschallexposition und späterem Autismus (autism spectrum disorders - ASD) fußt einzig und allein auf der Beobachtung, dass das Auftreten von Autismus in den letzten Jahrzehnten in etwa in gleichem Maße zugenommen hat wie die Anzahl an Ultraschalluntersuchungen. Andere Forschergruppen widersprechen dieser These und weisen darauf hin, dass sich im gleichen Zeitraum auch die Anzahl strahlungsintensiver Kommunikationsmittel (Mobiltelefone, W-LAN etc.) erhöht hat. In einer aktuellen Studie von Rosman et al. aus dem Jahr 2018 konnte gezeigt werden, dass bei den Müttern von 107 Kindern, die unter einem ASD litten (die Gesamtzahl der untersuchten Personen in dieser Studie betrug 211), weder häufiger Ultraschalluntersuchungen in der Schwangerschaft 
durchgeführt wurden, noch dass mit anderer Leistungseinstellung des Ultraschallgerätes untersucht worden war. Folglich muss das Auftreten von ASD nach dieser Studie als ultraschallunabhängig interpretiert werden.

Auch die manchmal als Beleg für Entwicklungsstörungen zitierte Arbeit von Ang et al. (2006) über eine gestörte NeuronenWanderung im Gehirn vom Mäuseföten nach Ultraschall-Exposition lässt nach Aussagen der Autoren keine Gefährdung für menschliche Föten erkennen. Insgesamt ist die Übertragung experimenteller Studien (Versuchsaufbau, Fixierung, Dauerbeschallung über einen langen Zeitraum, direkte Beschallung des Gehirns) auf den Menschen nur schwer möglich, da praktisch kein fetales Gehirn dauerhaft beschallt wird und im System Uterus, Fruchtwasser, Fötus und Mutter andere Bedingungen als im Tierexperiment bestehen.

\section{Kann der diagnostische Ultraschall sekun- däre Effekte in fetalen Organen hervorru- fen?}

\section{Antwort: Nein!}

Die aktuelle Studienlage gibt keine Hinweise auf fetale Zellveränderungen, Zellschädigungen und Kavitation nach Ultraschallexposition im üblichen Leistungsbereich. Laborversuche mit menschlichem Blut haben gezeigt, dass Kavitationseffekte (Entstehung von Gasbläschen durch Unterdruck) nur nach Anreicherung des Blutes mit Fremdmaterial auftreten und deshalb bei der Ultraschallexposition des Fötus ausgeschlossen werden können.

\section{Ist 3D/4 D-Ultraschall gefährlicher als 2D-Ultraschall?}

Nein, anders als vielerorts verlautet, führt die 3D/4D-Sonografie nicht zur höchsten, sondern - im Vergleich mit allen anderen Darstellungsmodi - zur geringsten thermischen Belastung für den Fötus. Ursache für diese besonders geringe Schallexposition sind die deutlichen längeren zeitlichen Intervalle zwischen jeweils 2 Abtastvorgängen.

Während die in der Schnittebene eines konventionellen 2D-Bildes liegenden Struktu- ren je nach gewähltem Maßstab und Anzahl der Fokusse etwa 20 - 50 mal pro Sekunde von den kurzen Ultraschall-Sendepulsen gestreift werden, sind die gleichen Strukturen im Falle einer volumetrischen Abtastung viel seltener, nur etwa 2-15 mal pro Sekunde, dem Ultraschall ausgesetzt. Diese geringe Abtastrate resultiert aus der $\mathrm{Ar}$ beitsweise heutiger Sonografie-Systeme, die das 3D-Volumen aus zahlreichen parallelen Einzelschnitten zusammensetzen. Diese Einzelschnitte entstehen jedoch nicht gleichzeitig, sondern werden seriell, also zeitlich nacheinander, aufgebaut!

Damit wird jeder Einzelort innerhalb des erfassten Volumens deutlich seltener von einem Ultraschallpuls getroffen als im Falle des 2D-Verfahrens oder gar bei Einsatz des Pulsdopplers. Da die thermische Belastung direkt vom sogenannten Taktverhältnis, also der Relation von der Dauer des Sendepulses (<1 1 sek) zur schallfreien Zeit (mehrere Millisekunden), bestimmt wird, nimmt die Belastung mit der Anzahl der Einzelschnitte und damit mit der Größe des 3D-Volumens ab.

Die ohnehin schon äußerst geringe Wahrscheinlichkeit etwaiger schädlicher Nebenwirkungen als Folge der konventionellen 2D-Abtastung wird damit bei Einsatz der $3 \mathrm{D} / 4 \mathrm{D}-$ Technik noch weiter reduziert!

Die DEGUM betont also die Ungefährlichkeit des Ultraschalls als Methode, wie sie ja auch in der Neufassung der Empfehlung „Orientierungshilfe für bildgebende Verfahren“ im Rahmen des Strahlenschutzgesetzes befürwortet wird. Dort wird der Ultraschall aufgrund seiner Ungefährlichkeit immer als primäres diagnostisches Mittel eingesetzt.

Provokant könnte man auch sagen, dann müssten auch jeder Schwangeren Flugreisen verboten werden, weil es dort dosis(Häufigkeit) und zeitdauerabhängig (Länge der Flugreise) in jedem Fall zu einer Exposition mit ionisierender Strahlung kommt, die bei entsprechender Dauer (EuropaUSA) einer Abdomen-Röntgen-Übersichtsaufnahme entspricht.

Somit unterstreichen die Autoren im Namen der DEGUM nochmal, dass es sich beim medizinischen Ultraschall nach dem derzeitigen Stand der Wissenschaft um ein Bildgebungsverfahren handelt, für das neben seiner hohen Aussagekraft Nebenwirkungen für Erwachsene, Kinder und Föten nicht nachgewiesen werden konnten. Entsprechend wird der Ultraschall auch in der Neufassung der Empfehlung „Orientierungshilfe für bildgebende Verfahren “ im Rahmen des Strahlenschutzgesetzes befürwortet und als primäres Bildgebungsverfahren empfohlen.

Auch hier besteht ein Arztvorbehalt, und es handelt sich in jedem Fall um medizinischen Ultraschall.

Eine Unterscheidung zwischen sogenanntem „Baby-Fernsehen“ und Ultraschall zur Beurteilung des Fötus in der Schwangerschaft ist schwierig. Die Methode, die verwendet wird, ist die gleiche. Man kann dem sogenannten „Baby-Fernsehen“ durchaus sehr kritisch gegenüberstehen, da sehr wohl die reale Gefahr besteht, tatsächliche Probleme des Fötus nicht zu erkennen. Sollte aber die Intention des Gesetzgebers sein, „Baby-Fernsehen“ zu verbieten, dann muss dazu der gesetzliche Ansatz ein anderer sein.

Die DEGUM steht als wissenschaftliche Fachgesellschaft für ein klar gegliedertes Qualitätskonzept mit verschiedenen Stufen und befürwortet uneingeschränkt die Anwendung des Ultraschalls zu diagnostischen Zwecken. Die in § 10 SSV geäußerten Bedenken hinsichtlich einer Gefährdung des Fötus durch Ultraschall sind in der Form nicht haltbar.

Für den Vorstand der DEGUM:

PD Dr. med. K.-S. Heling, Prof. Dr. med. P. Kozlowski und H. Dudwiesus Berlin, den 27. Februar 2019

Literatur

[1] Duggan PM, Liggins GC, Barnett SB. Ultrasonic heating of the brain of the fetal sheep in utero. Ultrasound Med Biol 1995; 21: 553-560

[2] Helmy S, Bader Y, Koch M et al. Measurement of Thermal Effects of Doppler Ultrasound: An In Vitro Study. PLoS ONE 2015; 10: e0135717. doi:10.1371/journal.pone.0135717 
[3] Rosman NP, Vassar R, Doros G et al. JAMA Pediatr 2018; 172: 336-344. doi:10.1001/jamapediatrics.2017.563

[4] Ang ES Jr, Gluncic V, Duque A et al. Prenatal exposure to ultrasound waves impacts neuronal migration in mice. Proc Natl Acad Sci USA 2006; 103: 12903 -12910. Epub 2006 Aug 10
[5] ISUOG Statement on ultrasound exposure in the first trimester and autism spectrum disorders 2016.

[6] EFSUMB Satement on the Safe Use of Doppler Ultrasound at 11 - 14 Weeks (or Earlier in Pregnancy) 2018.
[7] EFSUMB response to published study of the relationship between ultrasound exposure in the first trimester and the severity of autism spectrum disorders 2016.

[8] EFSUMB Clinicall Safety Statement for diagnostic Ultrasound 2018. 